\title{
THE WORST AVAILABILITY AS A PARAMETER FOR DESIGNING AND REPORTING ON THE NETWORK PERFORMANCES
}

\begin{abstract}
In the introductory part, the reasons why the availability is important are stated as well as the overview of the papers produced so far dealing with the problem of guaranteed availability. Furthermore, the basic concepts on availability are introduced with the special review of the parallel structure which can actually be applied onto the ring network. The principles of protection of the wavelength channel and optical multiplex section which are used in the ring WDM networks are described. Then the availabilities of different link configurations in relation to its protection are defined: without protection, protection using the same optical cable and protection using completely separated optical cables. Then the availability of the WDM ring was analysed which uses protection of the wavelength channel as well as the protection of the optical multiplex section. Furthermore, the expression for the worst availability of the WDM ring was derived and the number of links of the working path which gives the worst availability was determined. The data on the intensity of failures and the mean time to repair of individual components were taken from different literature. In the analysis we assumed that it is about WDM system with 64 wavelengths. At the end of the paper the answer to the question why the worst availability can be taken as a parameter at projecting WDM networks and for the reporting on the network performances was given.
\end{abstract}

Key words: WDM, wavelength channel, availability, protection.

\section{Introduction}

The development of photonic technologies enabled huge transmission speeds over optical fibres. The key technology for this development is WDM which enables hundreds of wavelength channels transmitting data in the $\mathrm{Gb} / \mathrm{s}$ speeds to be multiplexed in one signal suitable for transmission over optical cable. Exactly due to the large quantity of transmitted data, the network failures can cause big financial losses as well as the service provider's reputation. For that, applying different protection methods in WDM ring structure enables network operators to even, in the event of failures i.e. cable cut, provide to its customers/users normal work and qualitative service. Survival of the service is mostly represented through the availability of the connection which is a probability that the connection will be correct at some point in time. The connection availability depends on the availability of the network components along the path (optical fibres, optical add/drop multiplexors and switches...). In order to provide a quality service to its users, the operators apply different protection methods.

Defined availability in the SLA (Service Level Agreement) usually refers to the average availability of the connection [1]. However, the question is what the average availability means for the service user and if that is good enough to be protected against loss of incomes. If we assume, for example, that the average availability 0.995 in $99 \%$ cases, it means that still $1 \%$ of unavailability remains which for the big users who are transferring big data

\footnotetext{
* Ivan Rados ${ }^{1}$, Ladislav Schwartz ${ }^{2}$

${ }^{1}$ Department of Transmission Systems HT d.d., Mostar, Bosnia and Herzegovina, E-mail: Ivan.Rados@hteronet.ba

${ }^{2}$ Faculty of Electrical Engineering, University of Zilina, Slovakia
}

quantities (i.e. reda $\mathrm{Tb} / \mathrm{s}$ ) can mean significant loss of incomes and perturbation of the service quality.

In this paper we shall focus on the protection of the wavelength channel and optical multiplex section with 4 threads in the ring WDM network and by deriving the expressions for availability for both types of protection, we shall prove that the same expression is received in which only the availability of the optical link is changed depending on the protection we use.

We shall also introduce the term "the worst availability" and compare average and the worst availability in order to be able to conclude what big users should request from the operators through the SLA contract.

\section{Availability Theory}

where $G$ network consists of $N$ nodes and $N$ links connecting those nodes.

Availability $A_{s t}(G)$ between two terminals is a probability that there is at least one path which is composed of correct links and nodes between $s$ and $t$ in $G$.

If the nodes are ideal, then only $m=|E|$, remains as a subject to failure where each link $k=1,2, \ldots, m$ matches variable $x_{l k}$ and $\mathrm{x}$ represents a set of variables which represent links [2]: 


$$
\mathrm{x} \equiv\left(x_{l 1}, x_{l 2}, \ldots, x_{l m}\right)
$$

The aim is to calculate availability between two terminals and $t$ which is presented by a polynomial function of $\mathrm{x}$ elements having the characteristic that the availabilities of links $p_{l k}$ replace corresponding variables $x_{l k}$ which gives the numerical value which is exactly the availability between two terminals [3]

$$
A_{s t}(\mathrm{x})=A_{s t}\left(x_{l 1}, x_{l 2}, \ldots, x_{l m}\right)
$$

To complete the algebra formulation, two operators on polynomial will be introduced:

- $\otimes$, this operator is applied on the serial connection of two or more elements

- $\oplus$, this operator is applied on the parallel connection of two or more elements

Where $S$ represents a set of all polynomials which can come into existence by the combination of operators $\otimes$ and $\oplus$ so that the minimal value of the polynomial is 0 and the maximal is 1 . [9]:

For polynomials $f, g, h \in S$ following axioms are applicable

$$
\begin{array}{ll}
f \otimes f=f & f \oplus f=f \\
f \otimes 1=f & f \oplus 0=f \\
f \circledast 0=0 & f \oplus 1=1 \\
f \otimes g=g \otimes f & f \oplus g=g \oplus f
\end{array}
$$

If the operator $\otimes$ is applied on the serial structure, for instance of two elements whose failures are independent, the availability of such structure is equal to the product of availability of each single element.

$$
A s=x_{1} \otimes x_{2}=\prod_{l=1}^{2} x_{i}=x_{1} \cdot x_{2}
$$

It is generally applicable for the parallel structure of two elements

$$
A_{p}=x_{1} \oplus x_{2}=x_{1}+x_{2}-\left(x_{1} \otimes x_{2}\right)
$$

If the failures of elements are independent the following applies:

$$
A_{p}-x_{1} \oplus x_{2}=x_{1}+x_{2}-\left(x_{1}, x_{2}\right)
$$

If $x_{1}$ and $x_{2}$ are mutually exclusive which means that $x_{1} \otimes x_{2}=$ $=0$, we have

$$
A_{p}=x_{1} \oplus x_{2}=x_{1}+x_{2}
$$

To establish the connection between two nodes and network availability, we define $P_{s t}$ to consist of all simple paths $P_{i}$ between $s$ and $t$ in the network $G$
Also, we define the path value $v\left(P_{i}\right)$ by the "product" of the link variables along the path $P_{i}[2]$ :

$$
v\left(P_{i}\right)=\otimes\left\{x_{l k}: k \in P_{i}\right\}=\otimes \prod_{k \in P_{i}} x_{l k}
$$

The availability between two terminals $A_{s t}(\mathrm{x})$ is the "sum" of path value $v\left(P_{i}\right)$ taken over all the simple paths from $s$ to $t$.

$$
A_{s t}(\mathrm{X})=\oplus v\left(P_{i}\right), P_{i} \in P_{s t}=\oplus \sum_{P_{i} \in P_{s t}} v\left(P_{i}\right)
$$

The availability of certain elements (optical cables, nodes elements...) can be calculated by using the expression below

$$
A=\frac{M T T F}{M T T F+M T T R}
$$

Where MTTF (Mean Time to Failure) is mean time till the failure occurs and MTTR (Mean Time to Repair) mean time of repair [4].

$$
M T T F=1 / \lambda
$$

Where $\lambda$ is a failure rate which is usually expressed in FIT (Failure in Time, $1 F I T=1$ failure in $10^{9}$ hours).

Availability can be calculated on the basis of the collected data while the new systems use a probability model.

Unavailability $U$ is probability complementary to availability [12], i.e. $U=1-A$.

In reporting about the system/network performances, unavailability $U$ is often expressed as MDT (Mean Down Time) in minutes per year, i.e.

$$
M D T=365 * 60 * 24 * u \quad\left(\frac{\min }{g o d}\right)
$$

As an optical network generally consists of cable sections and nodes, an optical fibre failure rate is calculated separately from the node failure rate. The optical fibre failure rate is calculated according to the equation

$$
\lambda=\frac{n}{M * T}\left(\frac{l}{k m h}\right)
$$

Where $n$ is a number of failures over monitoring time, $M$ the length of installed cable in $\mathrm{km}$ and $T$ monitoring period in hours.

\section{Availability Analysis of WDM Ring}

Since the installation of more SDH line systems between two nodes is very expensive, as the capacities of optical cables exhaust considerably, the need for high transmission capacity system requiring only two fibers has arisen. Such are WDM systems based on wavelength multiplexing which use the wavelength channel protection and optical multiplex section protection. 
In order to derive the expression for the availability of the ring using mentioned protections, we shall define different types of links and nodes which are used thereat.

\subsection{Link availability}

In this paper we shall define three types of links in relation to their protection: without protection (unprotected), protection in the same cable and protection with totally separated cables [5] The unprotected optical link is comprised of optical cable and mux/demux as can be seen in Fig 1 .

All the components of the optical link must be correct so that the connection can be functional. The most often cause of an optical link failure is the breakage of optical fibre. Since, in the case of the cable breakage, mostly all the fibres break, we shall suppose that the failures of fibre and cable are fully dependent so that, instead of the availability for fibre we shall take the availability for cable.

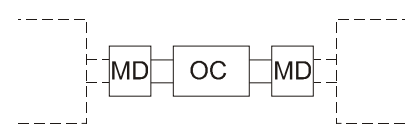

Fig. 1Unprotected link

The availability for link without the protection equals to the availability product of individual components.

$$
x_{l k_{n p}}=x_{O C} \cdot x_{M D}^{2}
$$

If optical interfaces are connected to fibres sharing the common cable (Fig. 2), the availability is:

$$
\begin{aligned}
& x_{l k_{\mathrm{K}}}=x_{w} \oplus x_{p}=x_{w}+x_{p}-x_{w} \otimes x_{p}= \\
& =x_{M D}^{2} \cdot x_{O C}+x_{M D}^{2} \cdot x_{O C}-\left(x_{M D}^{2} \cdot x_{O C} \otimes x_{M D}^{2} \cdot x_{O C}\right)= \\
& =2 x_{M D}^{2} \cdot x_{O C}-x_{M D}^{4} \cdot x_{O C}
\end{aligned}
$$

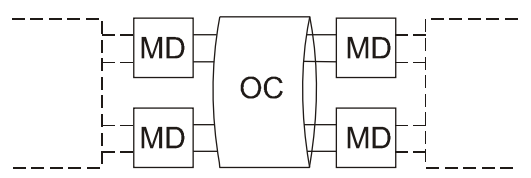

Fig. 2 A span-protected link consisting of two fiber pairs that share a common cable

Although there is product of $x_{O C} \oplus x_{O C}=x_{O C}$, only one member is taken for availability calculation because optical fibres share a common cable which means they have the same failure cause.

If optical interfaces are connected to the optical fibres belonging to completely separated cables (Fig. 3) availability is calculated as:

$$
\begin{aligned}
& x_{l k_{d c}}=x_{w} \oplus x_{p}=x_{w} \otimes x_{p}= \\
& =x_{M D}^{2} \cdot x_{O C_{1}}+x_{M D}^{2} \cdot x_{O C_{2}}-\left(x_{M D}^{2} \cdot x_{O C_{1}} \otimes x_{M D}^{2} \cdot x_{O C_{2}}\right)= \\
& =x_{M D}^{2} \cdot x_{O C_{1}}+x_{M D}^{2} \cdot x_{O C_{2}}-\left(x_{M D}^{4} \cdot x_{O C_{1}} \otimes x_{M D}^{2} \cdot x_{O C_{2}}\right)
\end{aligned}
$$

If the availability of both optical cables is equal, namely $x_{O C_{1}}=x_{O C_{2}}=x_{O C}$, the availability of the optical link with separated cables is

$$
\begin{aligned}
& x_{k_{d e}}=x_{w} \oplus x_{p}=x_{w}+x_{p}-x_{w} \otimes x_{p}= \\
& =x_{M D}^{2} \cdot x_{O C}+x_{M D}^{2} \cdot x_{O C}-\left(x_{M D}^{4} \cdot x_{O C} \cdot x_{O C}\right)= \\
& =2 x_{M D}^{2} \cdot x_{O C}-\left(x_{M D}^{4} \cdot x_{O C} \cdot x_{O C}\right)= \\
& =x_{M D}^{2} \cdot x_{O C} \cdot\left(2-x_{M D}^{4} \cdot x_{O C}\right)
\end{aligned}
$$

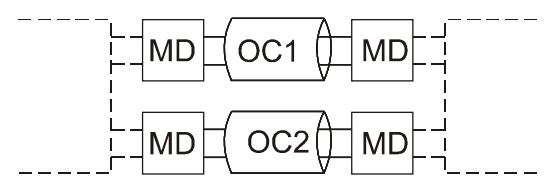

Fig. 3 A diversely-routed link consisting of two fibre pairs that are routed in diversity cables

\subsection{Node availability with active components}

Signals which are transported inside the ring are added/dropped from the nodes called "termination" nodes which are marked $s$ and $t$. Nodes in the ring through which the signal only passes from one side to the other are called "regeneration" nodes.

In accordance with that we shall define four node types depending on the trip of the signal through them [6]:

- terminal node is active, $x_{n t}$

- pass-through node is active, $x_{n p}$

- terminal node with the loop is active, $x_{n t l b}$

- pass-through node with the loop is active, $x_{n p l b}$

An optical switch with add/drop possibilities is used as the active component. In the case that the node is used as terminal, the wavelength channel passes through the transmitter, receiver and optical switch

$$
x_{n t}=x_{T X} \cdot x_{O S W} \cdot x_{R X}
$$

For the case of $n$ the wavelength channel in terminal node, the availability is

$$
x_{n t, n}=x_{T X}^{n} \cdot x_{O S W} \cdot x_{R X}^{n}
$$

If the node is a pass-through one the wavelength channel passes from east to west side of optical switch so that the availability is

$$
x_{n p}=x_{O S W}
$$


If the terminal node with the loop is active which is the case with the protection of the optical multiplex section, the wavelength channel passes through the transmitter, optical switch and receiver, the availability is

$$
x_{n t l b}=x_{T X} \cdot x_{O S W} \cdot x_{R X}
$$

But if the pass-through node with the loop is active, the wavelength channel only passes through the optical switch and the following applies:

$$
x_{n p l b}=x_{O S W}
$$

\subsection{The availability of the WDM ring with the wavelength channel protection}

This type of protection requires two fibres in a ring. Each wavelength channel is being routed on the working path along one side of the ring, and the corresponding dedicated wavelength channel, along the opposite side. Bidirectional wavelength requirements are supported by two wavelength channels; one in each direction.

Two types of dedicated protection are possible: $1+1$ and 1:1.

In the ring network which uses the $1+1$ wavelength channel protection the wavelength channel is the source node being duplicated and concurrently delivered in both directions of the ring [6];

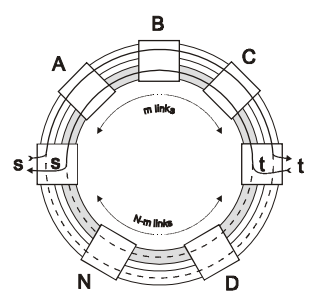

Fig. 4 1+1 wavelength channel protection under conditions without a failure

Under ordinary conditions in the terminal node, the receiver gets two signal copies (with a different delay) and chooses the best one. In the case of failure on the working path, the receiver chooses the signal that it gets from the protection path.

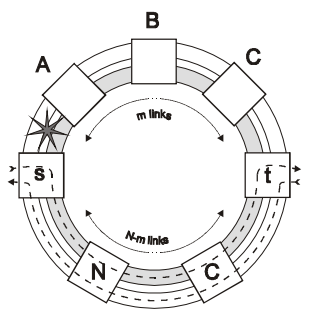

Fig. $51+1$ wavelength channel protection in the case of a failure on working path
This is so called single ended protection because the switching is carried out only on one (receiving) side. It is important that the working and protection paths do not have components in common, so that one failure would not cause total communication break down, and this means that the component failures on the working and protection paths are fully independent [7].

In 1:1 protection, the wavelength channel on the front side is not permanently duplicated so that the switching is performed dualended which requires a protocol to coordinate this dual-ended switching. From the availability point of view, the same availability expression applies as for the $1+1$ protection.

If a wavelength channel on the working path $P_{0}$ passes the $m$ of optical links between terminal nodes, the availability for the working path is equal to the availability product for optical links and nodes through which this wavelength channel passes [11]

$$
\begin{aligned}
& v_{s t}\left(P_{0}\right)=\otimes \prod_{k, m \in P_{0}} x_{l k} x_{n m}=\left[\otimes \prod_{k \in P_{0}} x_{l k}\right]\left[\otimes \prod_{k \in P_{0}} x_{n m}\right]= \\
& =\left(x_{n t}\right)^{2}\left(x_{n p}\right)^{m-1}\left[\otimes \prod_{k \in P_{0}}^{N-m} x_{l k}\right]
\end{aligned}
$$

In the case of failure on the working path, the wavelength channel passes the $N-m$ of optical links and the $N-m-1$ of nodes on the protection path $P_{1}$ so that the availability for the protection path is $(10)$

$$
\begin{aligned}
& v_{s t}\left(P_{1}\right)=\otimes \prod_{k, m \in P_{1}} x_{l k} x_{n m}=\left[\otimes \prod_{k \in P_{1}} x_{l k}\right]\left[\otimes \prod_{k \in P_{1}} x_{n m}\right]= \\
& =\left(x_{n t}\right)^{2}\left(x_{n p}\right)^{N-m-1}\left[\otimes \prod_{k \in P_{1}}^{N-m} x_{l k}\right]
\end{aligned}
$$

The availability for the wavelength channel between the $s$ and $t$ nodes is completely determined by these two paths so that the availability for the wavelength channel in the case of $1+1$ protection is calculated as the availability of two branches, failures of which are fully independent.

$$
\begin{aligned}
& A_{s t}(\times, m)=v_{s t}\left(P_{0}\right)+v_{s t}\left(P_{1}\right)-\left[v_{s t}\left(P_{0}\right) \otimes v_{s t}\left(P_{1}\right)\right] \\
& A_{s t}(\times, m)=\left(x_{n t}\right)^{2}\left(x_{n p}\right)^{m-1}\left[\otimes \prod_{k \in P_{0}}^{m} x_{l k}\right]+ \\
& +\left(x_{n t}\right)^{2}\left(x_{n p}\right)^{N-m-1}\left[\otimes \prod_{k \in P_{0}}^{N-m} x_{l k}\right]- \\
& -\left\{\begin{array}{c}
\left(x_{n t}\right)^{2}\left(x_{n p}\right)^{m-1}\left[\otimes \prod_{k \in P_{0}}^{m} x_{l k}\right] \\
\left.\otimes\left(x_{n t}\right)^{2}\left(x_{n p}\right)^{N-m-1}\left[\otimes \prod_{k \in P_{0}}^{N-m} x_{l k}\right]\right\}
\end{array}\right.
\end{aligned}
$$

Although in the equation brackets we have the product of the same two members, we use one member, not the square because of the earlier mentioned axioms which are applied for the operator $\otimes$; so we have

$$
\begin{aligned}
& A_{s t}(\times, m)=\left(x_{n t}\right)^{2}\left\{\left(x_{n p}\right)^{m-1}\left[\otimes \prod_{k \in P_{0}}^{m} x_{l k}\right]+\right. \\
& \left.+\left(x_{n p}\right)^{N-m-1}\left[\otimes \prod_{k \in P_{1}}^{N-m} x_{l k}\right]-\left(x_{n p}\right)^{N-2}\left[\otimes \prod_{k \in P_{0} P_{1}}^{N} x_{l k}\right]\right\}
\end{aligned}
$$

For the unprotected links [12] 


$$
\begin{aligned}
& A_{s t}(\times, m)=\left(x_{n t}\right)^{2}\left\{\left(x_{n p}\right)^{m-1}\left(x_{M D}\right)^{2 m}\left[\otimes \prod_{k \in P_{0}}^{m} x_{l k}\right]+\right. \\
& +\left(x_{n p}\right)^{N-m-1}\left(x_{M D}\right)^{2(N-m)}\left[\otimes \prod_{k \in P_{1}}^{N-m} x_{l k}\right]- \\
& \left.-\left(x_{n p}\right)^{N-2}\left(x_{M D}\right)^{2 N}\left[\otimes \prod_{k \in P_{0} \cdot P_{1}}^{N} x_{l k}\right]\right\}
\end{aligned}
$$

If we assume that optical links have the same length, their availability is the same, i.e.

$$
x_{l k}=x_{l}, \forall k
$$

In this case, the availability between the $s$ and $t$ nodes is

$$
\begin{aligned}
& A_{s t}(\times, m)=\left(x_{n t}\right)^{2}\left[\left(x_{n p}\right)^{m-1}\left(x_{M D}\right)^{2 m}\left(x_{l}\right)^{m}+\right. \\
& \left.+\left(x_{n p}\right)^{N-m-1}\left(x_{M D}\right)^{2(N-m)}\left(x_{l}\right)^{N-m}-\left(x_{n p}\right)^{N-2}\left(x_{M D}\right)^{2 N}\left(x_{l}\right)^{N}\right]
\end{aligned}
$$

\subsection{The availability of the WDM ring with optical multiplex section protection}

With this type of protection optical interfaces are connected on two or four optical threads which can be in the same cable or belong to the completely separated cables. We shall limit here to four, even though the same analysis stands for two threads only that the ring capacity in that case is twice smaller [8].

In the figure below the state of the optical ring with the protection of the optical multiplex section in case of no failure is presented. With OMS-SPRing-4 optical interfaces are connected to optical fibres sharing a common conduit and to those which are routed in separate conduits.

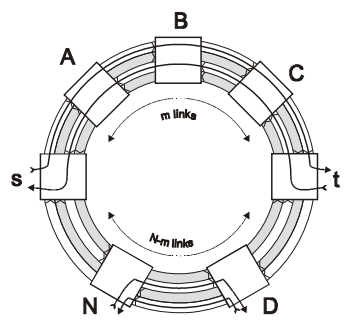

Fig. 6 WDM ring with optical multiplex section protection with 4 fibres under conditions without a failure

In a case of rupture of the optical cable, for instance between two "pass-through nodes", as a reaction to the failure the loop is formed in them and the signal is redirected to other two protection threads [9].

In addition to the working path there are several restoration paths with OMS-SPRing 4, depending on the failure place. All the failures, single or multiple, owing to which the transport signal inside the ring can be restored in less than 60 ms need to be considered in this analysis. If we suppose that there are $x$ failures, availability of OMS-SPRing 4 can be written as

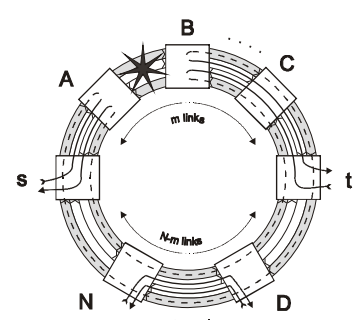

Fig. 7 WDM ring with optical multiplex section protection with 4 fibres in the case of a failure on working path

$$
A_{s t}(\times, m)=v_{s t}\left(P_{0}\right) \oplus\left[\oplus \sum_{i=1}^{x} v_{s t}\left(P_{1}\right)\right]
$$

Where $v_{s t}\left(P_{0}\right)$ is working path availability, $v_{s t}\left(P_{1}\right)$ is availability of one of $x$ restoration paths and the mark $\oplus$ represents "sum". Although the number of failures which can be restored can be high it can be demonstrated that the "sum "of all the restoration paths reduced to one path, which means:

$$
v_{s t}\left(P_{A m}\right)=\left[\oplus \sum_{i=1}^{x} v_{s t}\left(P_{1}\right)\right]
$$

Where $v_{S t}\left(P_{A m}\right)$ is the availability of the worst restoration path $P_{m}$ which is the case when all the working path links and nodes are faulty. If we put the availability of the worst restoration path into the expression for OMS-SPRing 4, we obtain

$$
A_{s t}(\times, m)=v_{s t}\left(P_{0}\right) \oplus v_{s t}\left(P_{A m}\right)
$$

Whereat

$$
v_{s t}\left(P_{A m}\right)=v_{s t}\left(P_{1}\right)
$$

which means that the same expression is obtained for the availability between two terminals using protection of the wavelength channel. Detailed analyses can be found in [10]

\section{Worst Terminal Pair Availability}

In order to obtain the worst availability regarding the number of links, it is necessary to derive this expression for availability under $m$

$$
\begin{aligned}
& A_{s t}(\times, m)=\left(x_{n t}\right)^{2}\left[\left(x_{n p}\right)^{m-1}\left(x_{M D}\right)^{2 m}\left(x_{l}\right)^{m}+\right. \\
& \left.+\left(x_{n p}\right)^{N-m-1}\left(x_{M D}\right)^{2(N-m)}\left(x_{l}\right)^{N-m}-\left(x_{n p}\right)^{N-2}\left(x_{M D}\right)^{2 N}\left(x_{l}\right)^{N}\right]
\end{aligned}
$$

When equation (32) derive by $\mathrm{m}$ and equalise to zero, than

$$
\begin{aligned}
& \frac{d}{d m}\left[\left(x_{n p}\right)^{m-1}\left(x_{M D}\right)^{2 m}\left(x_{l}\right)^{m}+\right. \\
& \left.+\left(x_{n p}\right)^{N-m-1}\left(x_{M D}\right)^{2(N-m)}\left(x_{l}\right)^{N-m}-\left(x_{n p}\right)^{N-2}\left(x_{M D}\right)^{2 N}\left(x_{l}\right)^{N}\right]=0
\end{aligned}
$$

After simple derivation we obtain the expression 


$$
\begin{aligned}
& {\left[\left(x_{n p}\right)^{m-1}\left(x_{M D}\right)^{2 m}\left(x_{l}\right)^{m}+\left(x_{n p}\right)^{N-m-1}\left(x_{M D}\right)^{2(N-m)}\left(x_{l}\right)^{N-m}\right.} \\
& \left.\cdot m\left(x_{n p}\right)^{-L}+2 m\left(x_{M D}\right)^{-1}+m\left(x_{l}\right)^{-1}\right]=0
\end{aligned}
$$

After arranging we obtain

$$
\begin{aligned}
& m-1=N-m-1, \Rightarrow m=N / 2 \\
& 2 m=2(N-m), \Rightarrow m=N / 2 \\
& m=N-m, \Rightarrow m=N / 2
\end{aligned}
$$

This is valid for even number. Since $m$ has to be whole number, this expression cannot be applied for uneven number $N$ and the following is used

$$
m=\frac{N-1}{2}
$$

Examine is about the minimum or maximum. We have to determine second derivation of this expression and determine whether it is $>0$ or $<0$

$$
\begin{aligned}
& {\left[\left(x_{n p}\right)^{m-1}\left(x_{M D}\right)^{2 m}\left(x_{l}\right)^{m}+\left(x_{n p}\right)^{N-m-1}\left(x_{M D}\right)^{2(N-m)}\left(x_{l}\right)^{N-m}\right.} \\
& \left.\cdot m\left(x_{n p}\right)^{-L}+2 m\left(x_{M D}\right)^{-1}+m\left(x_{l}\right)^{-1}\right]=0
\end{aligned}
$$

By simple calculation, it is possible to demonstrate that the second derivation is $>0$ and that it is about the minimum or, in other words, the worst availability which is obtained for $m=N / 2$ for even number of nodes and

$$
m=\frac{N-1}{2}
$$

for odd number of nodes.

\section{Numerical Results}

In order to calculate the availability for a WDM system we need to know the nodes and optical links availability. For availability calculation, in general, one needs to know the intensity of failures for the individual components, the data of which are shown in Table 1, and are taken from different literature and published works [6].

Availability data for optical components $(\mathrm{W}=64)$

Table 1

\begin{tabular}{|l|c|c|}
\hline Component/Device & Symbol & Failure rate rarateRate(FIT) \\
\hline Line Amplifier & LOA & 3200 \\
\hline Multiplexer & MUX & $25 \times \mathrm{W}$ \\
\hline Demultiplexer & DEMUX & $25 \mathrm{xW}$ \\
\hline Optical Switch & OSW & 1000 \\
\hline Fix Transmitter & TX & 186 \\
\hline Fix Receiver & RX & 70 \\
\hline Cable $($ per km) & OC & 100 \\
\hline
\end{tabular}

NOTE: $W$ is the number of wavelength channels
MDT (min/year) for a node with active components

Table 2

\begin{tabular}{|c|c|}
\hline & MDT \\
\hline MTTR $=6 \mathrm{~h}$ & $\lambda=64$ \\
\hline Terminal & 3.22 \\
\hline Pass-through & 0.000018 \\
\hline
\end{tabular}

We shall calculate the availability for different number of nodes, from 6 to 10 which is a common size of rings in realistic networks and equal link length of $20 \mathrm{~km}$. For MTTR equipment and cables 6 and 12 hours is taken, which is also common in practice.

\section{$\mathrm{N}=6, \mathrm{~d}=20 \mathrm{~km}$}

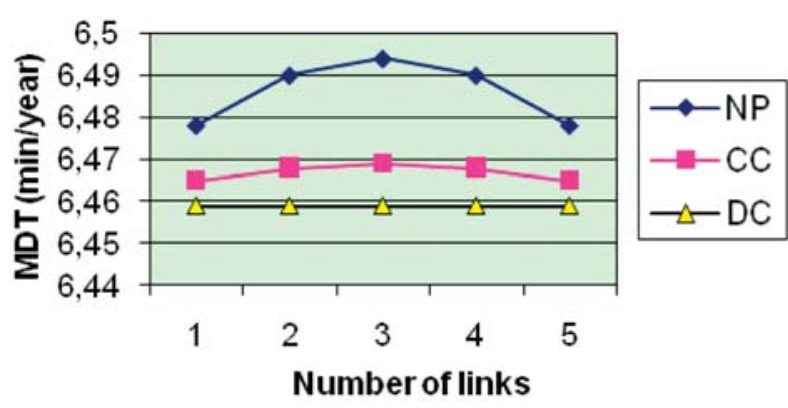

Fig. $8 \operatorname{MDT}(\mathrm{min} /$ year $)$ for $N=11$ and $d=20 \mathrm{~km}$

\section{$\mathrm{N}=8, \mathrm{~d}=\mathbf{2 0} \mathrm{km}$}

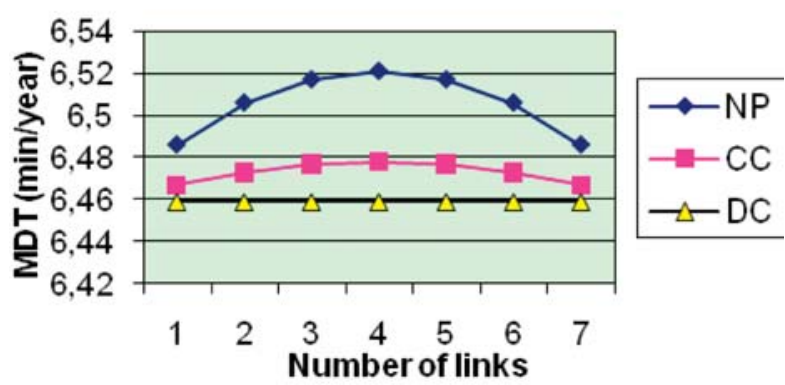

Fig. $9 \operatorname{MDT}(\mathrm{min} /$ year $)$ for $N=12$ and $d=20 \mathrm{~km}$

$\mathrm{N}=10, \mathrm{~d}=20 \mathrm{~km}$

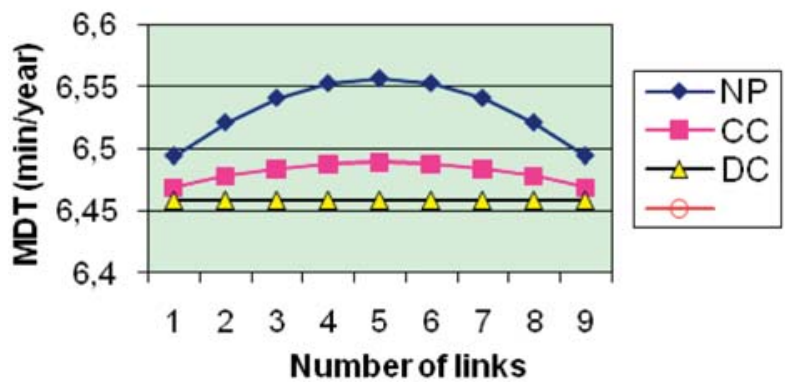

Fig. $10 \mathrm{MDT}$ (min/year) for $N=12$ and $d=60 \mathrm{~km}$ 
As can be seen in the previous graphs, the obtained worst availability expressed over MDT is in accordance with the earlier derived expression for the number of links of the working path, so for example, for the ring with different number of nodes which is unprotected, we have:

- $N=6, m=N / 2=3$, MDT $=6.494$

- $N=8, m=N / 2=4, \mathrm{MDT}=6.521$

- $N=10, m=N / 2=5$, MDT $=6.557$

It can also be seen that with the increment of the number of nodes, unavailability is increased with the same length of links. Also, by applying protection, the number of unavailable minutes per year is decreased: i.e. for $N=6$, MDT for the case of protection through the same cable in comparison with the case without protection is decreased by $0.38 \%$ and for the case of totally separated cables, the difference is increased to $0.54 \%$. The difference between the worst availability and the availability which is obtained for the certain number of links of the working path is decreased by applying protection: i.e. if we observe MDT for $N=8$ unprotected, the difference between the worst availability which is in this case for 4 links of the working path and availability for 1 link of the working path is $0.035 \mathrm{~min} /$ year while for the case of protection through the same cable that difference is $0.011 \mathrm{~min} /$ year and for the case of totally separated cables, the difference is zeromeaning that the availability is always equal, notwithstanding the number of links of the working path. Also this difference is increasing by the increment of the number of nodes, so if we suppose the same length of links for the ring-unprotected, the biggest difference from $0.063 \mathrm{~min} /$ year $(N=10), 0.035 \mathrm{~min} /$ year $(N=8)$ to $0.016 \mathrm{~min} /$ year $(N=6)$. For the cases with protection those differences are even smaller. Therefore, we can conclude that all the availabilities between two terminals, notwithstanding the number of links of the working path, are approximately equal for a given ring and that the availability between two terminals for a given ring notwithstanding protection can be presented through the worst availability.

\section{Conclusion}

The analysis of the results on different WDM rings shows that the worst availability is obtained in accordance with the earlier derived expression for the number of links of the working path and that is $m=N / 2$ for the even number of links and $m=N-1 / 2$ for uneven number of links of the working path. It is also to note that with the premise of the same length of links, the unavailability is increasing with the increment of the number of nodes in the ring. Also by applying different methods of protection, the unavailability in relation to the case without protection is decreasing and it is the smallest in the case when totally separated cables are used for protection. Due to small differences between the worst availability and the availability between two terminals notwithstanding the number of links of the working path presuming that the links are of the same length, the availability between two terminals can generally be presented through the worst availability and as such can be used as a parameter when projecting new or when reporting on the performances of the existing networks.

\section{References}

[1] MELlO, A.A., PELEGRINI, J.U., RIBERIO, R.P., SCHUPKE, D.A., WALDMAN, H.: Dynamic Provisioning of Shared-Backup Path Protected Connections with Guaranteed Availability Requirements, International Workshop on Guaranteed Optical Service Provisioning, Boston, USA, 2005

[2] DOUGLAS, S.R.: Network Reliability and Algebraic Structures, Oxford University Press : New York, 1991,

[3 ] JONCZY, J.: An algebraic Approach to Network Reliability, Institute of Computer Science and Applied Mathematics, University of Berne, 2008

[4] TRSTENSKY, D., SCHWARTZ, L., TRUNKVALTER, M., BAROS, R.: Reliability of Telecommunication System by Various Repair Regime, Int. Conference ELEKTRO 2004, Advances in Electrical and Electronic Engineering, No. 2, Vol. 3/2004, ZU, Zilina, pp. 25-26, ISSN 1336-1376

[5] WILSON, M.R.: The Quantitative Impact of Survivable Network Architecture on Service Availability, IEEE Communications Magazine, May 1998, pp.122-127,

[6] RADOS, I.: Availability Models for Wavelength Channel Protection in WDM Ring Networks, J. of Electronics and Electrical Engineering, No. 8(88), 2008, pp.81-86, Kaunas University of Technology

[7] MELLO, A.A., QUITERIO, G.S., SCHUPKE, D.A., WALDMAN, H.: Specification of SLA Survivability Requirements for Optical Path Protected Connections, Optical Fiber Communications Conference (OFC), Anaheim, 2006,

[8] ZHANG, J., ZHU, Z., ZANG, H., MUKHERJEE, M.: Service Provisioning to Provide Per-Connection-based Availability-Guarantee in WDM Mesh Networks, Optical Communications Conference (OFC), 2003,

[9] MYKKELTVEIT, A., HELVIK, B.E.: On Provision of Availability Guarantees Using Shared Protection, Optical Network Desing and Modeling (ONDM), 2008,

[10] RADOS, I.: Availability of the optical bidirectional self-healing ring with four fibres with the Multiplex Section ProtectionAvailability, SoftCOM 2008, Split. 